\title{
Optical and IR Observations of Aql X-1
}

\author{
Michael R. Garcia and Paul J. Callanan \\ Smithsonian Astrophysical Observatory, Cambridge, MA, USA
}

\begin{abstract}
We have obtained optical spectra and IR photometry in an attempt to determine the orbital parameters of the Aql X-1 system. Spectra from the MMT in October 1995 appear to detect the secondary, but are not sufficient to measure the mass function. IR photometry in and out of mini-outburst find $K \sim 15.4$, and may indicate that the disk contribution at $K$ is modest during the mini-outburst.
\end{abstract}

\section{Introduction}

Aql X-1 is a Low-Mass X-ray Binary (LMXB), containing a neutron star primary, which undergoes irregular outbursts approximately once per year. During the outbursts, which may last for 1-2 months, the optical counterpart reaches $V \sim 17$ (mini-outbursts), and occasionally $V \sim 15$. Photometry obtained during an extended mini-outburst shows a modulation at $18.97 \pm 0.02$ hours, which may be the orbital period (Chevalier and Ilovaisky 1991).

\section{Optical Spectra}

In October 1995 we obtained a series of spectra covering the $5500 \AA-9000 \AA$ range at $3.6 \AA$ resolution with the MMT, when the system was near quiescence. The MMT TV guider image ( $\sim R$-band) and photometry before and after the MMT run indicate $V=19 \pm 0.2$. We cross-correlated the $5600 \AA-6800 \AA$ data against a variety of templates, avoiding $30 \AA$ regions round the $5890 \AA, 6300 \AA, 6370 \AA$ (night-sky lines), and $\mathrm{H} \alpha$. The strongest correlations we found for mid-K spectral types. A doppler-corrected sum of the 9 spectra with the strongest correlations (180 min total exposure) is shown in Figure 1. Many of the photospheric lines in the template (HD6660, K4V) are visible in the Aql X-1 spectrum, in particular the lines at $6495 \AA, 6160 \AA, \mathrm{NaD}$, etc. We do not detect the Li $6708 \AA$ line seen in V404 Cyg, but out upper limit of $E W \sim 0.3 \AA$ is similar to the strength of the line found in V404 Cyg (Martin et al. 1992). The number and phase distribution of spectra is not sufficient to allow the mass function to be measured.

The $\mathrm{H} \alpha$ line is clearly double peaked, with a $E W=-4.3 \AA$, and $\mathrm{FWHM}=$ $740 \mathrm{~km} / \mathrm{sec}$. This is rather narrow, and may indicate a 'face-on' inclination. The relation between line width and inclination found for CVs and Black-Hole X-ray Binaries, $F W H M=1340 \sin (i) *(M / P)^{1 / 3}$, (Garcia et al. 1996) predicts $i=20^{\circ} \pm 10$, assuming $M=1.4 M_{\text {sol }}$ and $P=0.79$ days. 


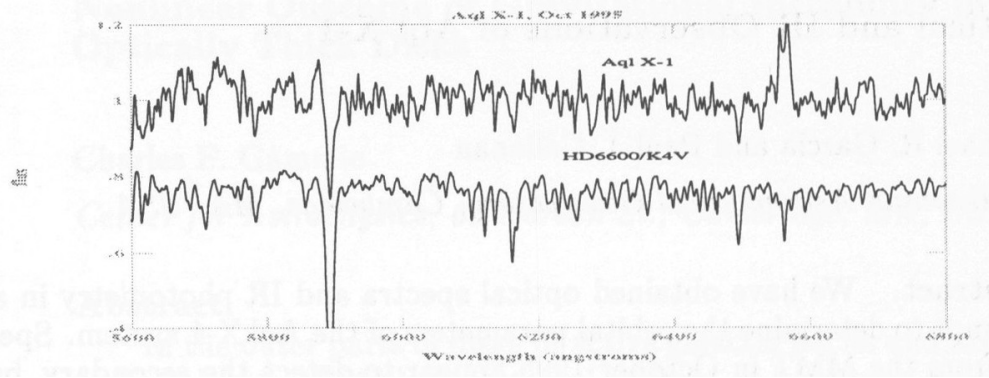

Figure 1. Spectrum of Aql X-1 in Oct 1995

\section{IR and Optical Photometry}

Infrared photometry from the Steward $61^{\prime \prime}$ found $K_{s}=15.4 \pm 0.3$ on March 16 , 1996. Photometry from the Whipple Observatory $48^{\prime \prime}$ a few days later found $V=18.85 \pm 0.03$, indicating that the system was near quiescence. A few months later (June/July 1996) the system was in mini-outburst, with $K_{s}=15.14 \pm 0.03$, $V=16.87 \pm 0.03$, and a count rate of $\sim 3 c / s$ in the XTE/ASM.

During the mini-outburst the source brightens at $K_{s}$ by only $0.26 \pm 0.3 \mathrm{mag}$; indeed it may have remained constant. The one sigma upper limit to the disk contribution during mini-outburst is 0.56 . In the optical, the light from LMXB is dominated by the disk during outburst, but it appears that in the IR the disk and secondary contributions may be nearly equal during this mini-outburst.

Previous estimates of the companions spectral type have found G7-K3 (Thorstensen etal 1978), or G8-K0 (van Paradijs 1996). We can estimate the spectral type from our $\mathrm{V}$ and $\mathrm{K}$ photometry obtained in quiescence. The reddening has been previously estimated (Thorstensen 1978) at $E(B-V)=0.4$, which indicates a de-reddened $V_{0}=17.8 \pm 0.2$. If we assume that the disc contributes $\sim 50 \%$ of the light at $\mathrm{V}$, as is found in several quiescent LMXB, we find an intrinsic $V_{0, s e c}=18.3 \pm 0.2$. This yields a $V-K=2.9 \pm 0.4$, which corresponds to the color of K1 - K4 giants or K4 - K6 dwarfs (Johnson 1966). This spectral range is slightly later than that found in previous works.

\section{References}

C. Chevalier and S.A. Ilovaisky 1991 A\&A 251, L11

Garcia, M.R, Callanan, P.J, Zhao, P., McClintock, J.E., 1996, in 'Cataclysmic Variables and Related Objects', ed Evans and Wood, Kluwer.

Martin, AL, Rebolo, R., Casares, J, and Charles, P.A. 1992, Nature, 358129.

Thorstensen, J, Charles, P, and Bowyer, S, 1978 ApJ 220, L131.

van Paradijs, J., 1996 in "X-ray Binaries", ed. Lewin, van Paradijs, and van den Heuvel, CUP.

Johnson, HL, 1966 Ann. Rev. Ap. 4, 193. 